\title{
Automatic identification of epileptic EEG signals through binary magnetic optimization algorithms
}

\author{
Luís A. M. Pereira ${ }^{1}$ João P. Papa ${ }^{2}$ - André L. V. Coelho ${ }^{3}$ Clodoaldo A. M. Lima ${ }^{4}$ \\ Danillo R. Pereira ${ }^{2}$ - Victor Hugo C. de Albuquerque ${ }^{3}$
}

Received: 2 November 2016/Accepted: 17 June 2017/Published online: 28 June 2017

(C) The Natural Computing Applications Forum 2017

\begin{abstract}
Epilepsy is a class of chronic neurological disorders characterized by transient and unexpected electrical disturbances of the brain. The automated analysis of the electroencephalogram (EEG) signal can be instrumental for the proper diagnosis of this mental condition. This work presents a systematic assessment of the performance of different variants of the binary magnetic optimization algorithm (BMOA), two of which are introduced here, while serving as feature selectors for epileptic EEG signal identification. In this context, the optimum-path forest classifier was adopted as a classification model, whereas different wavelet families were considered for EEG feature extraction. In order to
\end{abstract}

João P. Papa

papa@fc.unesp.br

Luís A. M. Pereira

luismartinspr@gmail.com

André L. V. Coelho

acoelho.albuquerque@unifor.br

Clodoaldo A. M. Lima

c.lima@usp.br

Danillo R. Pereira

danilopereira@unoeste.br

Victor Hugo C. de Albuquerque

victor.albuquerque@unifor.br

1 Instituto de Computação, Universidade Estadual de Campinas, Campinas, SP, Brazil

2 Departamento de Computação, UNESP - Univ Estadual Paulista, Bauru, SP, Brazil

3 Programa de Pós-Graduação em Informática Aplicada, Universidade de Fortaleza, Fortaleza, CE, Brazil

4 Escola de Artes, Ciências e Humanidades, Universidade de São Paulo, São Paulo, SP, Brazil compare the performance of the improved BMOA variants against the traditional one, as well as other metaheuristic techniques, namely particle swarm optimization, binary bat algorithm, and genetic algorithm, we employed a well-known EEG benchmark dataset composed of five classes of EEG signals (two of which comprising normal patients with eyes open or closed, and the remaining comprising ill patients with different levels of epilepsy). Overall, the results evidenced the robustness of the proposed BMOA and its variants.

Keywords Feature selection · Epilepsy · EEG signal classification - Magnetic optimization algorithm . Metaheuristics · Optimum-path forest

\section{Introduction}

Broadly speaking, epilepsy can be defined as a medical condition related to the occurrence of seizures, which affect a variety of mental and physical functions of an individual. In short, the term "epilepsy" encompasses a number of different neurological syndromes characterized by transient and unexpected electrical disturbances of the brain [4]. In epileptic patients, the brain's normal electrical activity is disrupted by overactive electrical discharges, causing a temporary communication problem among nerve cells [3]. It is estimated that epilepsy is the third most common neurological disorder in the USA, being around 50-65 million people worldwide affected by such class of syndrome. Besides, the mortality rate is two to three times higher among people with epilepsy, which is fair enough for increasing the investments on novel methodologies and computational devices for the early and correct diagnosis of this medical condition. 
One of the most reliable examinations for the proper diagnosis of seizures and epilepsy is the well-known electroencephalogram (EEG) [3], which records the brain's electrical activity as a series of traces, each of them corresponding to a different region of the brain. However, the visual inspection of the EEG signals for the detection of normal, interictal, and ictal activities in the patient's brain is usually a time-consuming and error-prone task due to the huge volumes of EEG segments that have to be analyzed. Therefore, the adoption of computer-based techniques for the purpose of tackling epilepsy diagnosis via EEG signal classification has been actively pursued in the last decades [8]. Besides, since EEG signals are nonlinear and dynamic in nature [1], there has been a growing interest in applying nonlinear signal analysis techniques, such as those based on wavelets, entropy, fractal, and chaos theory [37], for studying the behavior of these signals and also to extract relevant and condition-discriminatory information from them.

In order to assess the pros and cons of different machine learning approaches to cope with the epilepsy diagnosis problem, several prominent works have recently employed different configurations of the EEG dataset made available by Andrzejak et al. [1, 2]. This benchmark dataset is composed of five classes in total (two of which comprising normal patients with eyes open or closed, and the remaining comprising ill patients with different levels of epilepsy), whose full discrimination is very hard to achieve. In this context, Subasi [26-28] employed some variants of artificial neural networks (ANN) and also mixture-of-experts (ME) models aiming to discriminate between seizure and seizure-free profiles. In [27], in particular, the author reported $94.5 \%$ of accuracy rate achieved by ME models while discriminating solely classes $A$ and E, which was a score better than that achieved by single multilayer perceptron (MLP) neural networks $(93.2 \%)$. The specificity and sensitivity values reported for the ME and MLP models were, respectively, 94\%/92.6\% and $95 \% / 93.6 \%$. ME models induced with wavelet coefficients have also been considered by Übeyli [33], even though, in that work, the performance of the models was measured over three sets of the EEG dataset (namely sets A, D, and E). The total classification accuracy achieved by the ME network structures was $93.17 \%$ [33].

On the other hand, the paper of Tzallas et al. [31, 32] presents a methodology whereby selected segments of the EEG signals (maybe with different sizes) are analyzed using time-frequency methods, and then several features are extracted for each segment representing the energy distribution in the time-frequency plane. These features are used as input to a feedforward neural network, which provides the final classification. In order to evaluate the methodology, the authors generated four different classification problems, none of which, however, involving the five classes at the same time, and the results achieved in terms of overall accuracy ranged from 97.72 to $100 \%$. Nunes et al. [21] carried out a simple application of the optimum-path forest classifier [23, 24] to diagnose patients with epilepsy via EEG signal classification using four types of wavelet functions for feature extraction, being the Coiflets as the most accurate ones.

Lima et al. [14-16] evaluated the potentials of several kernel-based learning machines, such as support vector machines (SVM) and relevance vector machines (RVM), in the task of automatic discrimination of epileptic from nonepileptic EEG signals. The performance levels obtained by the kernel machines were contrasted in terms of predictive accuracy, sensitivity to the kernel function/parameter value, and sensitivity to the type of features extracted from the signal. For this purpose, several types of features extracted from the EEG signal, including statistical values derived from the discrete wavelet transform, Lyapunov exponents, and combinations thereof, were considered. Overall, the results evidenced that all considered kernel machines were competitive in terms of accuracy, and the choice of the kernel function and parameter value, as well as the choice of the feature extractor, are really critical decisions to be taken into account.

In this paper, we focus our attention on one specific step of the whole classification process that was not deeply investigated in the aforementioned works, i.e., the step of selecting the optimal subset of discriminatory features extracted from the EEG signal. In a nutshell, feature selection, also known as variable or attribute selection, is the task of selecting a subset of relevant features for inducing a classifier model [9, 10]. The central assumption when using a feature selection technique is that the data contain many redundant or irrelevant features. While redundant features are those which provide no more information than the currently selected features, irrelevant features provide no useful information at all. Even though the theme of feature selection has been much researched in the last years, it is noticeable that only a few works have given some attention to the study of the impact of this step in the context of EEG signal classification.

The paper of Ocak [22], for instance, is an exception, where the use of a genetic algorithm-based (GA) EEG feature selector was investigated. In the proposed scheme, normal and epileptic EEG segments were decomposed into various frequency bands through a wavelet packet decomposition. Then, approximate entropy values of the wavelet coefficients at all nodes of the decomposition tree were used as candidate features to characterize the predictability of the EEG data within the corresponding frequency bands. Finally, the GA was used to find the subset of features that maximizes the classification performance 
of an EEG classifier based on learning vector quantization (LVQ). It was particularly demonstrated in [22] that, if the GA was not used for the optimal feature selection, the good classification accuracies achieved by the LVQ classifier would drop noticeably.

In this paper, our emphasis is on the investigation of the potentials of a recently introduced population-based metaheuristic technique, named as magnetic optimization algorithm (MOA) [30], to serve as selector of optimal EEG features extracted by different wavelet families. Since the feature selection task is computationally intractable for even moderate sizes of feature sets [9, 10], the analysis of the performance of different metaheuristic algorithms for performing this task is readily justified [39]. Moreover, since the feature selection task can be regarded as a binary optimization problem, different variants of the binary magnetic optimization algorithm (BMOA) [18] have been considered in this study, two of which are introduced here. We compared MOA-based algorithms against GA, particle swarm optimization (PSO) [17, 38], and binary bat algorithm (BBA) [19], being the experiments conducted over the aforementioned EEG benchmark dataset.

The remainder of the paper is organized as follows. In Sect. 2, we outline the main steps behind the BMOA variants considered. Section 3 formalizes the steps of the proposed feature selection methodology, while Sect. 4 characterizes the EEG dataset and the wavelet basis used as feature extractors. Section 5 presents how the computational experiments were set up, while Sect. 6 is devoted to assess the performance of the techniques for EEG signal classification, taking into account the impact of the different feature selectors. Finally, Sect. 7 states conclusions.

\section{Magnetic optimization algorithm}

The electromagnetic force concept is one of the four fundamental interaction forces in nature. In this interaction force, the force intensity concerning two electromagnetic particles is inversely proportional to the distance between them, i.e., the greater the distance, the smaller the interaction force. Based on this definition, Tayarani and Akbarzadeh [30] proposed a new metaheuristic algorithm called magnetic optimization algorithm (MOA), which models a system of magnetic particles (agents) that seek for a solution in a search space using their magnetic fields, i.e., their fitness values, to interact with each other. The mathematical definitions of MOA are summarized as follows:

- Initially, MOA starts randomly placing all agents in the search space. Each agent is modeled as a solution vector $\mathbf{x}_{i} \in \mathfrak{R}^{\mathrm{D}}$, where $i$ denotes the $i$-th agent, and $x_{i}^{d}$ stands for its position at $d$-th dimension.

- At each iteration of the algorithm, the solution vectors are evaluated, and their respective fitness values are stored in $B_{i}$, which denotes the magnetic field value of the particle $i$.

- The mass $M_{i}$ of each agent is given by:

$M_{i}=\alpha+\rho B_{i}$,

where $\alpha$ and $\rho$ are constant parameter values.

- The interaction force between two particles $i$ and $j$ at dimension $d$ is given as follows:

$F_{i j}^{d}=B_{i} \frac{x_{j}^{d}-x_{i}^{d}}{D\left(\mathbf{x}_{\mathbf{j}}, \mathbf{x}_{\mathbf{i}}\right)}$,

in which $D(\cdot, \cdot)$ is a distance function.

- The acceleration, velocity, and the position of each agent are updated, respectively, by:

$a_{i}^{d}=\frac{F_{i}^{d}}{M_{i}}$,
$v_{i}^{d}(t+1)=\theta v_{i}^{d}(t)+a_{i}^{d}$

and

$x_{i}^{d}(t+1)=x_{i}^{d}(t)+v i^{d}$,

where $t$ is the iteration step and $\theta \sim U(0,1)$.

Tayarani and Akbarzadeh [30] also proposed a lattice where each agent can be influenced by the magnetic field from its neighborhood, being possible to determine the total force acting over each particle. However, this sort of lattice provides low and limited interactions, since an agent can interact with its immediate neighbors only (four neighborhoods) [18].

\subsection{Binary MOA}

Mirjalili and Hashim [18] proposed a binary version of the original MOA (BMOA) aiming to tackle binary optimization problems. In addition, they introduced a fully connected topology, in which all particles are connected and can interact to each other, thereby improving the shortcomings of the four-neighborhood lattice topology. Hereafter, we will refer to BMOA configured with a fourneighborhood lattice as $\mathrm{BMOA}_{1}$, whereas $\mathrm{BMOA}_{2}$ refers to the one with fully connected topology.

In order to restrict the new particle's position to only binary values, the authors employed a hyperbolic tangent function [18]:

$S\left(v_{i}^{d}(t)\right)=\left|\tanh \left(v_{i}^{d}(t)\right)\right|$. 


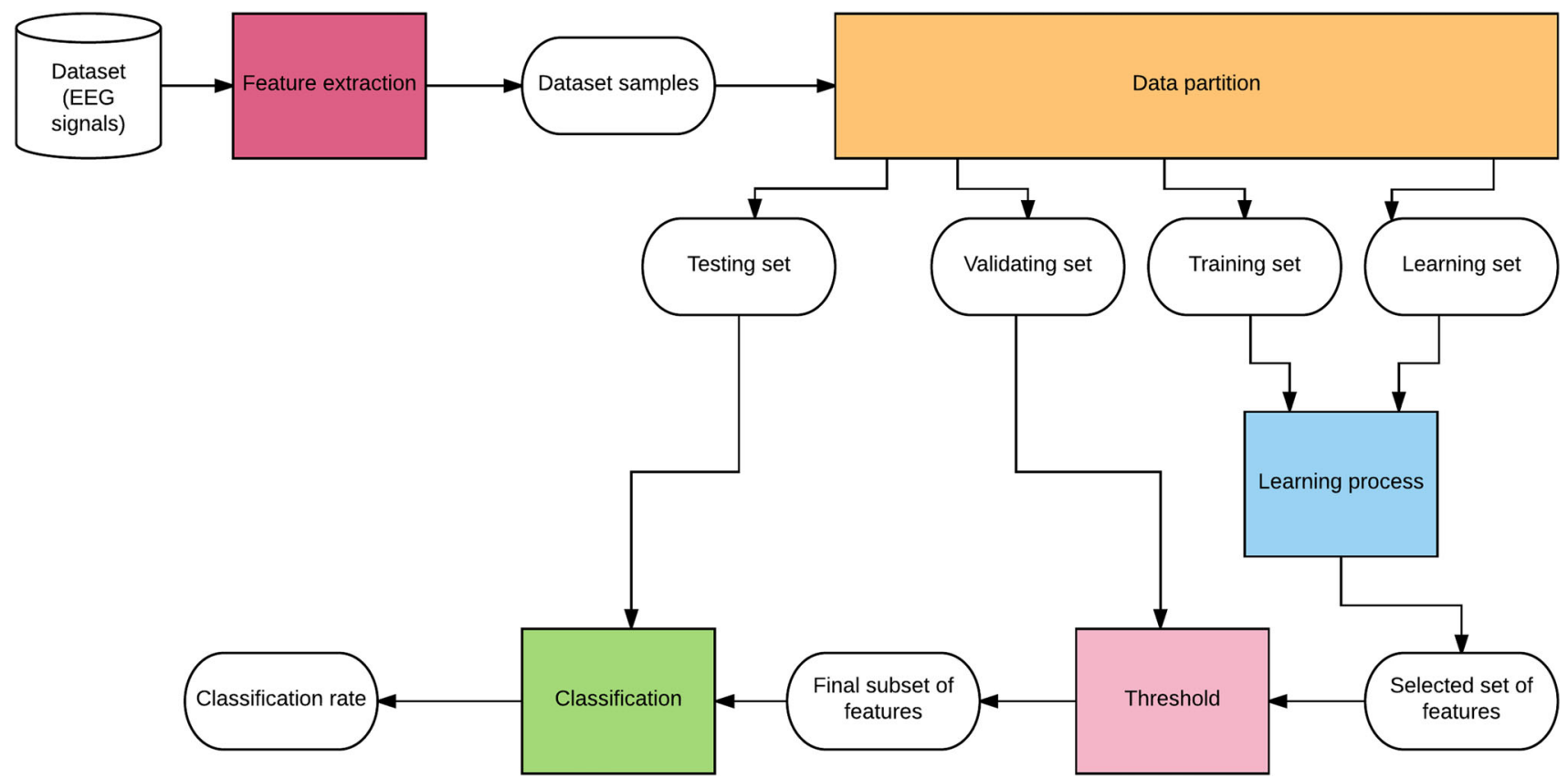

Fig. 1 Pipeline of the proposed feature selection methodology

Equation (6) can be rewritten as:

$x_{i}^{d}(t+1)=\left\{\begin{array}{cl}\neg\left(x_{i}^{d}(t)\right) & \text { if } S\left(v_{i}^{d}(t+1)\right)>\sigma, \\ x_{i}^{d}(t) & \text { otherwise }\end{array}\right.$

in which $\neg(\cdot)$ means the binary complement operator and $\sigma \sim U(0,1)$. To provide a good convergence rate, the velocity was limited to $\left|v_{i}^{d}(t+1)\right|<v_{\max }$, where $v_{\max }$ was set to 6 .

\subsection{Improving BMOA}

Although $\mathrm{BMOA}_{2}$ has demonstrated more interaction benefits than $\mathrm{BMOA}_{1}$, the particles suffer from the attraction of bad ones, which may cause the loss of the previous good solution. As such, we propose here two new variants of the BMOA algorithm:

1. In the first variant, named as $\mathrm{BMOA}_{3}$, we model the interaction between good and bad particles, so that only particles with a good magnetic field can attract particles with bad magnetic ones. Therefore, a particle $i$ with a magnetic field $B_{i}$ will attract a particle $j$ only if $B_{i}>B_{j}$ (in case of maximization problems). Thus, the resultant force $F_{j}$ over a bad particle $j$ is formed by the attraction from particles with better magnetic fields than $B_{j}$.

2. The second variant, called $\mathrm{BMOA}_{4}$, uses the same interaction strategy employed by $\mathrm{BMOA}_{3}$; however, it allows that some good particles be attracted by some bad ones, as follows:
$\frac{B_{j}-\text { best } B}{B_{j}-B_{i}}>\sigma$ or $B_{j}>B_{i}$.

However, these conditions may introduce the same problem as in $\mathrm{BMOA}_{2}$, i.e., losing good solutions. In order to avoid this problem, we introduce a vector $\mathbf{y}$ to store the best local position of each particle $i$. Thus, we update $\mathbf{y}_{i}$ only if the new solution $\mathbf{x}_{i}(t+1)$ achieves a better solution. These procedures are similar to those presented in [12], but for a different approach.

\section{Feature selection methodology}

In this section, we present the methodology used to evaluate the proposed variants of BMOA. The main idea is to allow a fair unbiased mean recognition rate computation together with a proper subset of suitable features. In order to accomplish with such deals, let us introduce some important definitions. Let $\mathcal{Z}$ be a labeled dataset, such that $\mathcal{Z}=\mathcal{Z}_{1} \cup \mathcal{Z}_{2} \cup \mathcal{Z}_{3} \cup \mathcal{Z}_{4}$, in which $\mathcal{Z}_{1}, \mathcal{Z}_{2}, \mathcal{Z}_{3}$, and $\mathcal{Z}_{4}$ stand for the training, learning, validating, and test sets, respectively. Roughly speaking, the main goal of a metaheuristic-based feature selection approach is to employ some classifier's recognition rate to be part of the fitness function (wrapper approaches). In this work, we use the training and learning sets to guide the search process onto the solution space ("Learning process" module in Fig. 1). Therefore, the idea is to train a classifier over $\mathcal{Z}_{1}$ for further 
classification of $\mathcal{Z}_{2}$ (for each search agent), being the recognition rate over the latter set used as the fitness function. As one can realize, we need a fast and effective classifier, since we need to perform the training step followed by the classification of the learning set every time an agent changes its position. Therefore, we opted to employ the supervised optimum-path forest (OPF) classifier [23, 24], which is a parameter-free technique that has been used for several applications.

The above procedure, which outputs the selected subset of features that maximizes the OPF accuracy over $\mathcal{Z}_{2}$, is then conducted 10 times with randomly generated training and learning sets. Thus, one has at the final of the process, 10 subsets of selected features, being now the main goal to choose the best one. Such step is conducted by the "Threshold" module in Fig. 1: we employed a thresholdbased approach to find out the final subset of features, being such threshold value ranged from 10 to $90 \%$, with steps of $10 \%$. A threshold value of $T \%$, for instance, means we selected the features that appeared at least $T \%$ on that 10 subsets outputted over the 10 executions of the "Learning process" module in Fig. 1. The selected features for that threshold, i.e., $T \%$, are then used to train OPF for further classification of the validating set $\left(\mathcal{Z}_{3}\right)$. Therefore, if we perform the above procedure for each threshold within the range $[10 \%, 90 \%]$, we obtain a curve that represents the recognition rate over $\mathcal{Z}_{3}$ for each threshold value. The final subset of features is the one which maximizes the accuracy of such curve, being such subset used to train OPF for further classification of the unseen testing set

Table 1 Parameter setting of the metaheuristic algorithms

\begin{tabular}{ll}
\hline Technique & Parameters \\
\hline BBA & $\alpha=0.9, \gamma=0.9$ \\
BGA & $p m=0.1$ \\
BMOA & $\alpha=0.9, \rho=4.8$ \\
BPSO & $c_{1}=2.0, c_{2}=2.0, w=0.9$ \\
\hline
\end{tabular}

$\left(\mathcal{Z}_{4}\right)$. Notice the test set has not been used so far, i.e., it has been employed for assessing the effectiveness of the final subset of features only.

Figure 1 illustrates our proposed methodology to select the subset of features that best represents EEG signals. We used $30 \%$ of the original dataset for $\mathcal{Z}_{1}, 20 \%$ for $\mathcal{Z}_{2}, 20 \%$ for $\mathcal{Z}_{3}$, and $30 \%$ for $\mathcal{Z}_{4}$. These percentages were set up empirically.

We also compared the MOA-based approaches against a mutual information (MI) filter-based method [20]. The best MI model was chosen by selecting the features percentage among $[10 \%, 20 \%, \ldots, 90 \%]$ with the highest mutual information. For this purpose, $\mathcal{Z}_{1}$ was employed as the training set and $\mathcal{Z}_{2} \cup \mathcal{Z}_{3}$ as the validating sets. Thereafter, the OPF classifier was trained on $\mathcal{Z}_{1}$ to classify $\mathcal{Z}_{4}$.

\section{Dataset description}

In this work, we evaluate the performance of BMOA and its variants in the context of automatic epilepsy diagnosis. The complete dataset consists of five sets (denoted as AE), which contains 100 single-channel EEG segments of 23.6s. These segments were selected and cut out from continuous multi-channel EEG recordings after visual inspection for artifacts due to muscle activity or eye movements. All EEG signals were recorded with the same 128-channel amplifier system using an average common reference. The data were digitized at $173.61 \mathrm{~Hz}$ sampling rate with 12 bit analog-to-digital resolution, and the bandpass filter settings were $0.5340 \mathrm{~Hz}(12 \mathrm{~dB} / \mathrm{oct})$. The data are made available by Andrzejak et al. [1, 2].

The signals from folds A and B were obtained extracranially from surface EEG recordings of five healthy individuals with eyes open and closed, respectively. Notice the sets C, D, and E were originated from an EEG archive of pre-surgical diagnosis. The EEG signals from five patients were selected, all of whom had achieved complete seizure control after resection of one of the hippocampal
Table 2 Mean recognition rates considering OPF over the original (baseline) testing set

\begin{tabular}{|c|c|c|c|c|c|c|c|c|c|c|c|c|c|c|c|c|}
\hline & \multirow[t]{2}{*}{ Accuracy $(\%)$} & \multicolumn{5}{|c|}{ F-measure } & \multicolumn{5}{|c|}{ Precision } & \multicolumn{5}{|c|}{ Recall } \\
\hline & & A & B & $\mathrm{C}$ & $\mathrm{D}$ & $\mathrm{E}$ & A & B & $\mathrm{C}$ & $\mathrm{D}$ & $\mathrm{E}$ & A & B & $\mathrm{C}$ & $\mathrm{D}$ & $\mathrm{E}$ \\
\hline Coif2 & 64 & 63 & 70 & 55 & 39 & 92 & 55 & 74 & 57 & 42 & 93 & 73 & 67 & 53 & 37 & 90 \\
\hline Coif3 & 70 & 70 & 74 & 61 & 56 & 91 & 64 & 72 & 65 & 55 & 100 & 77 & 77 & 57 & 57 & 83 \\
\hline Coif4 & 68 & 58 & 74 & 67 & 45 & 95 & 56 & 78 & 59 & 52 & 97 & 60 & 70 & 77 & 40 & 93 \\
\hline $\mathrm{Db} 2$ & 61 & 69 & 60 & 43 & 41 & 85 & 65 & 70 & 42 & 46 & 76 & 73 & 53 & 43 & 37 & 97 \\
\hline Db3 & 60 & 65 & 51 & 46 & 48 & 87 & 56 & 71 & 48 & 45 & 84 & 77 & 40 & 43 & 50 & 90 \\
\hline $\mathrm{Db} 4$ & 60 & 72 & 51 & 59 & 42 & 71 & 64 & 62 & 47 & 56 & 86 & 83 & 43 & 80 & 33 & 60 \\
\hline Sym2 & 62 & 73 & 64 & 42 & 46 & 84 & 61 & 74 & 44 & 45 & 92 & 90 & 57 & 40 & 47 & 77 \\
\hline Sym3 & 59 & 66 & 58 & 41 & 48 & 81 & 65 & 64 & 41 & 44 & 83 & 67 & 53 & 40 & 53 & 80 \\
\hline Sym4 & 59 & 72 & 52 & 54 & 33 & 84 & 65 & 58 & 48 & 38 & 92 & 80 & 47 & 63 & 30 & 77 \\
\hline
\end{tabular}


Table 3 Mean recognition rates and number of selected features considering BMOA variants over the testing set

\begin{tabular}{|c|c|c|c|c|c|c|c|c|}
\hline & \multicolumn{2}{|l|}{$\mathrm{BMOA}_{1}$} & \multicolumn{2}{|l|}{$\mathrm{BMOA}_{2}$} & \multicolumn{2}{|l|}{$\mathrm{BMOA}_{3}$} & \multicolumn{2}{|l|}{$\mathrm{BMOA}_{4}$} \\
\hline & $\operatorname{Acc}(\%)$ & \#Selected features & $\operatorname{Acc}(\%)$ & \#Selected features & $\operatorname{Acc}(\%)$ & \#Selected features & $\operatorname{Acc}(\%)$ & \#Selected features \\
\hline Coif2 & 71 & 16 & 71 & 24 & 69 & 39 & 69 & 20 \\
\hline Coif3 & 70 & 7 & 72 & 11 & 75 & 18 & 81 & 13 \\
\hline Coif4 & 76 & 31 & 71 & 4 & 76 & 34 & 75 & 24 \\
\hline $\mathrm{Db} 2$ & 67 & 24 & 61 & 22 & 73 & 8 & 67 & 10 \\
\hline Db3 & 69 & 11 & 73 & 11 & 69 & 12 & 69 & 15 \\
\hline Db4 & 69 & 16 & 72 & 16 & 71 & 26 & 72 & 14 \\
\hline Sym2 & 66 & 8 & 62 & 7 & 68 & 4 & 71 & 6 \\
\hline Sym3 & 67 & 14 & 58 & 20 & 61 & 22 & 69 & 10 \\
\hline Sym4 & 66 & 12 & 67 & 16 & 67 & 10 & 67 & 22 \\
\hline
\end{tabular}

Bold values indicate the most accurate techniques

Table 4 Mean recognition rates and number of selected features considering BBA, BGA, BPSO, and MI on test set

\begin{tabular}{|c|c|c|c|c|c|c|c|c|}
\hline & \multicolumn{2}{|l|}{ BBA } & \multicolumn{2}{|l|}{ BGA } & \multicolumn{2}{|l|}{ BPSO } & \multicolumn{2}{|l|}{ MI } \\
\hline & $\operatorname{Acc}(\%)$ & \#Selected features & $\operatorname{Acc}(\%)$ & \#Selected features & $\operatorname{Acc}(\%)$ & \#Selected features & $\operatorname{Acc}(\%)$ & \#Selected features \\
\hline Coif2 & 69 & 20 & 72 & 16 & 66 & 18 & 72 & 32 \\
\hline Coif3 & 72 & 11 & 78 & 22 & 80 & 24 & 80 & 28 \\
\hline Coif4 & 78 & 10 & 76 & 33 & 75 & 31 & 72 & 16 \\
\hline $\mathrm{Db} 2$ & 69 & 33 & 67 & 6 & 67 & 15 & 64 & 8 \\
\hline Db3 & 64 & 25 & 75 & 16 & 66 & 15 & 68 & 8 \\
\hline Db4 & 64 & 4 & 68 & 15 & 68 & 8 & 60 & 8 \\
\hline Sym2 & 63 & 14 & 64 & 15 & 68 & 4 & 66 & 8 \\
\hline Sym3 & 65 & 14 & 54 & 21 & 69 & 11 & 62 & 16 \\
\hline Sym4 & 67 & 6 & 66 & 14 & 64 & 16 & 65 & 8 \\
\hline
\end{tabular}

Bold values indicate the most accurate techniques

formations, which was therefore correctly diagnosed to be the epileptogenic zone. Signals in the folds C and D were sampled intracranially in seizure-free intervals from five patients. While the signals in fold $\mathrm{C}$ were captured from the hippocampal formation of the opposite hemisphere of the brain, those from fold D were extracted directly from the epileptogenic zone. Finally, fold $\mathrm{E}$ contains signals obtained intracranially and related to the seizure activity. These signals were selected from all recording sites of the brain exhibiting ictal activity. We consider here the whole dataset of 500 EEG segments, each one with 4096 samples, as employed in [34].

\section{Experimental design}

This section describes the main steps involved in our experimental procedures. We compared the MOA-based approaches against three other feature selection techniques: binary bat algorithm (BBA) [25], binary particle swarm optimization algorithm (BPSO) [6], and binary genetic algorithm (BGA) [13].

- Parameter setting: Table 1 presents the parameters employed for each metaheuristic technique. Notice we used 30 agents with 100 iterations for all techniques. These parameters were set based on previous experiments.

- Statistical evaluation: In order to give more support for our conclusions, we carried out two round of statistical tests. Firstly, we performed the nonparametric Friedman test, which was used to rank the algorithms for each dataset separately. In case of Friedman test to provide meaningful results to reject the null-hypothesis $\left(h_{0}\right.$ : all techniques are equivalent), then we can perform a post hoc test. For this purpose, we perform the Nemenyi test [20], which allows us to verify whether there is a critical difference (CD) among techniques. The results of the Nemenyi test can be represented in a simple diagram, in which the average ranks of the methods are plotted on an horizontal axis, 


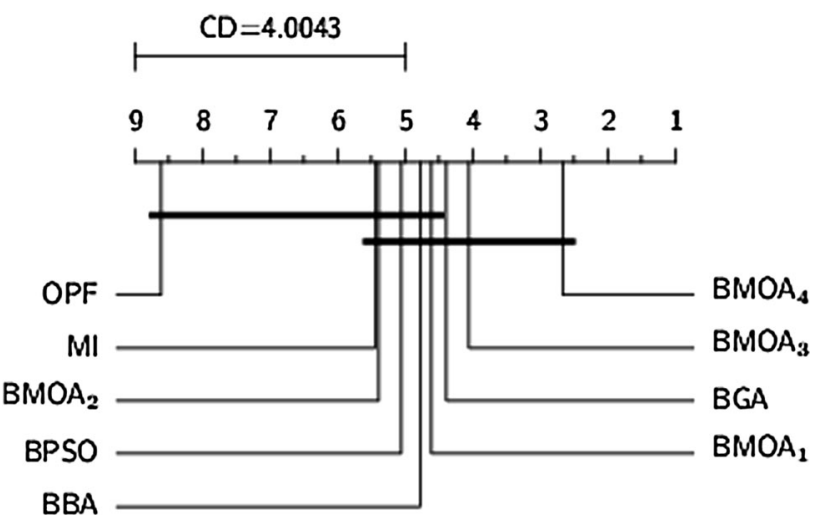

Fig. 2 Nemenyi statistical test considering the accuracy results

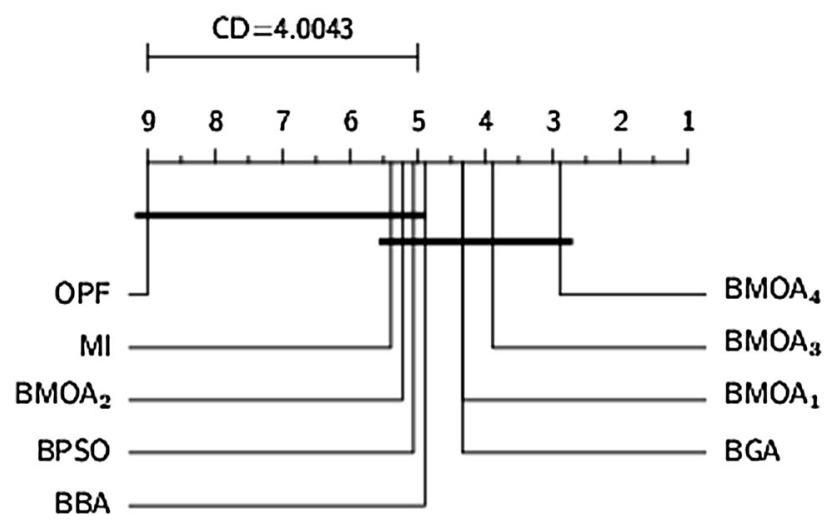

Fig. 3 Nemenyi statistical test considering the F-measure results

where the lower average rank is better. Furthermore, the groups with no significantly difference are then connected. More about these procedures can be found in Demšar [5].

- Performance measures: in order to assess the performance of the feature selection techniques, four wellknown measures were employed: standard accuracy, F-measure, precision, and recall. Since we are dealing with a problem with multiple classes, the three latter measures were calculated for each class separately.

- Fitness function: as the reader may have noticed, our methodology (Sect. 3) requires a fast training and classification steps. In this fashion, we employed the OPF classifier, since it is a nonparametric and very robust classifier. Thus, for each iteration of the optimization techniques, the fitness function is calculated as the accuracy [24] of the OPF classifier on the learning set.

- Platform: it is important to highlight that all experiments were carried out on a PC Intel ${ }^{\circledR}$ Core i7 Q740 $1.73 \mathrm{GHz}$ with $3 \mathrm{~GB}$ RAM running Ubuntu 10.04 as operational system.

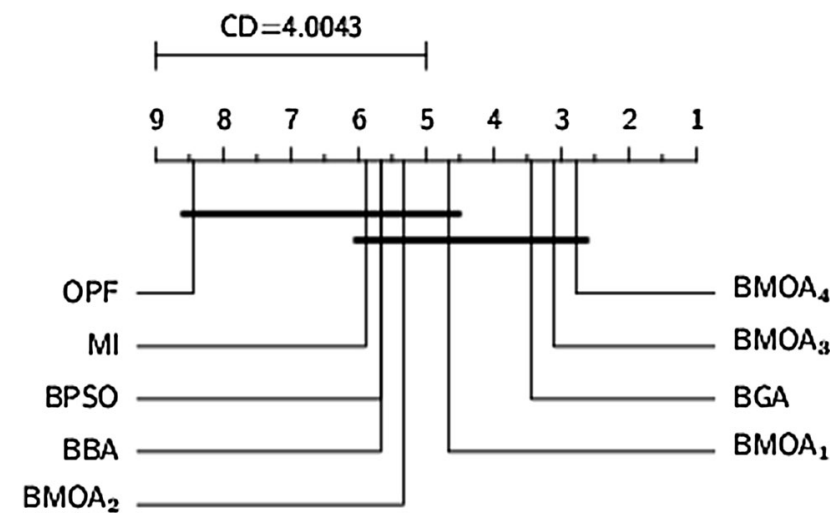

Fig. 4 Nemenyi statistical test considering the precision results

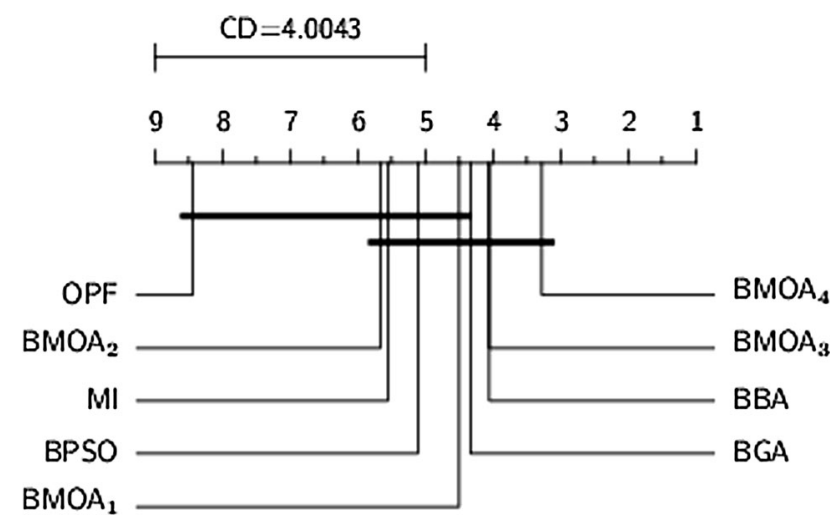

Fig. 5 Nemenyi statistical test considering the recall results

In order to extract discriminatory features from raw EEG data, the discrete wavelet transform (DWT) was employed in this work $[29,36]$. The basic idea underlying wavelet analysis consists in expressing a signal as a linear combination of a set of localized functions, which are obtained by shifting, contracting, and dilating one particular prototype function, called a mother wavelet [11]. The decomposition of the signal leads to a set of values, referred to as wavelet coefficients.

While conducting the experiments for this paper, we have also considered different wavelet families with different orders and parametrization factors. However, due to the lack of space, we focus our analysis here on the Coiflets (Coif) order 2-4, the Symlet (Sym) order 2-4, and Daubechies $(\mathrm{Db})$ order $2-4[7,14]$. Therefore, 40 feature values were extracted from each of the 500 data patterns available in the dataset. The chosen features are related to the well-known statistics calculated over the wavelet coefficients in each or adjacent sub-bands, i.e., minimum, maximum, mean, standard deviation, power, absolute mean, and ratio of absolute mean [14, 27, 33, 35]. 
(a)

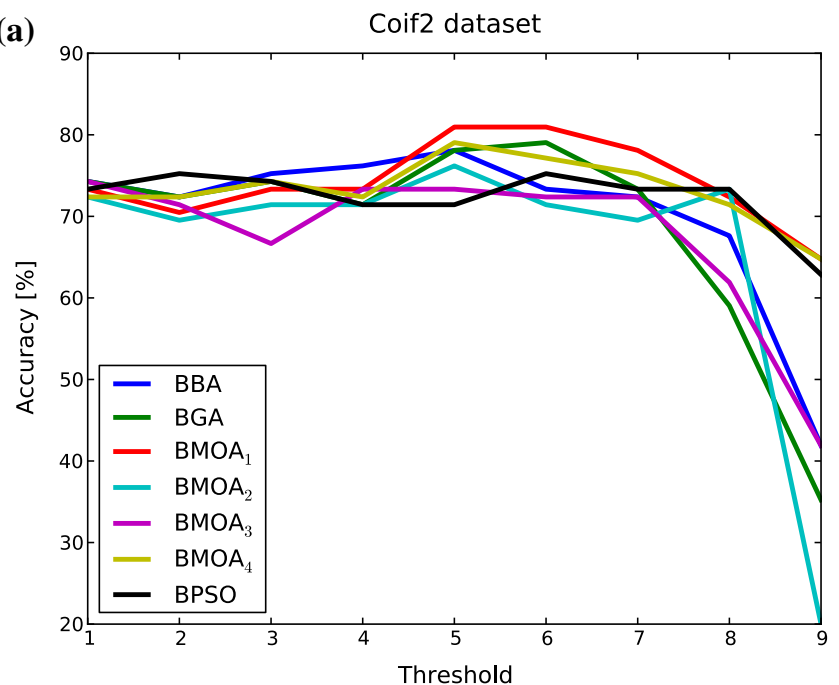

(c)

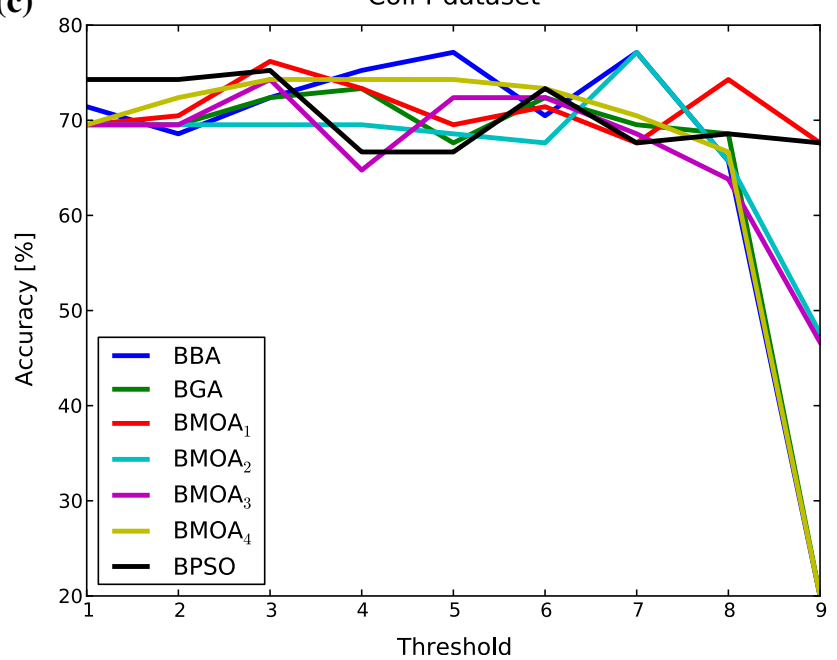

(b)

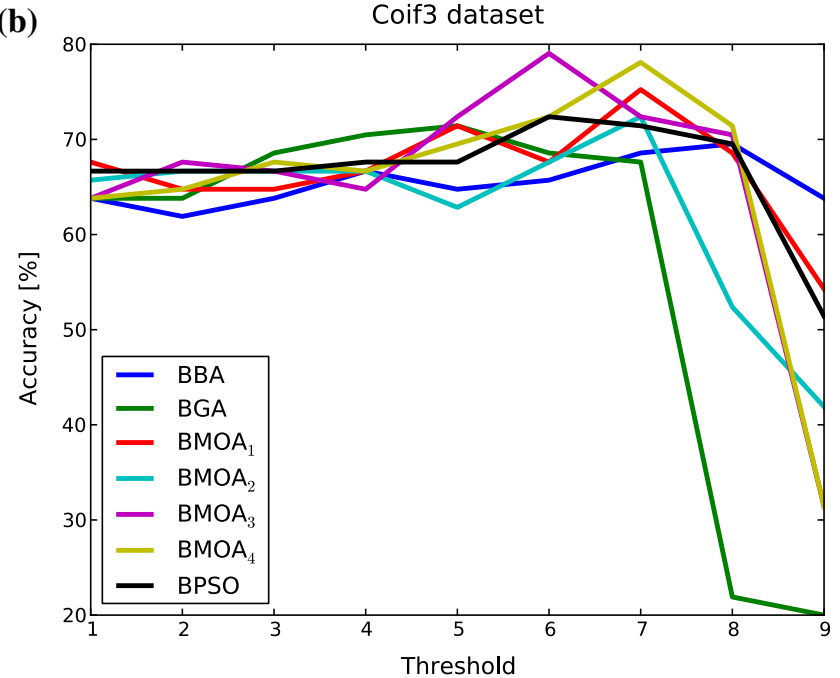

(d)

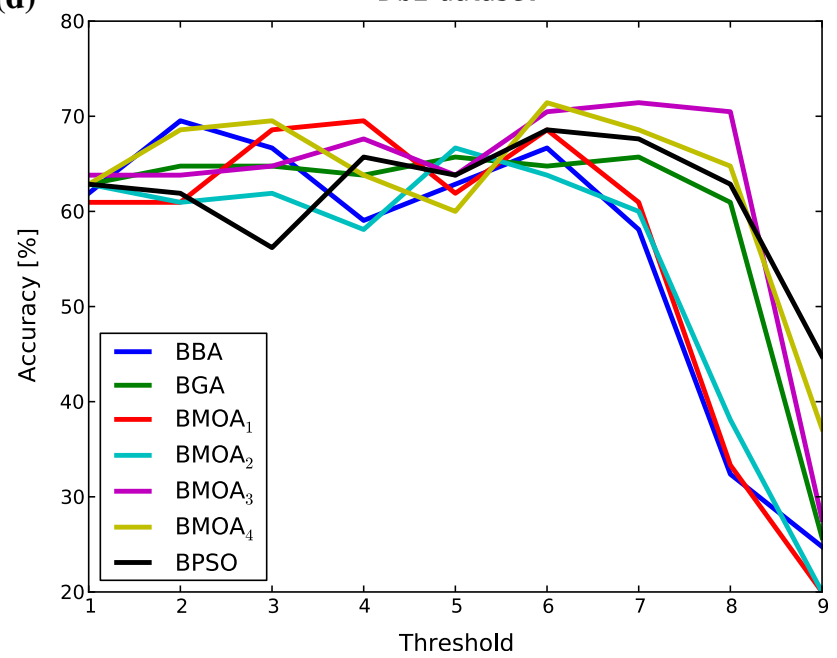

Fig. 6 Accuracy rates over the validating set considering Coif2, Coif3, Coif4, and Db2 datasets

\section{Results and discussion}

In this section, we present the results obtained using the proposed approaches. In order to provide a baseline for comparison purposes, we evaluated the performance of OPF classifier over the original datasets, i.e., without feature selection. For such experiment, we employed only the training and testing sets, since the learning and validating sets were used for feature selection purposes (Sect. 3). Notice the training and test sets were the same as the ones used in the feature learning process.

Table 2 shows the OPF classifier results over the original datasets (baseline), as well as Tables 3 and 4 display the recognition rates concerning the feature selection approaches. Notice that improvements on the accuracies after feature selection for all datasets can be observed. In regard to the Coif3 dataset, for instance, $\mathrm{BMOA}_{1}$ achieved the same results as the OPF classifier, but it has selected only seven features. The same behavior can be observed for $\mathrm{Db} 2$ and Sym2 datasets, in which $\mathrm{BMOA}_{2}$ presented the same OPF results, but it has selected 22 and 7 features, respectively. If we consider the Sym 3 dataset, $\mathrm{BMOA}_{2}$ was the only technique that did not surpass the performance of the OPF classifier.

Figures 6 and 7 depict the curves generated by the "Threshold" module described in Fig. 1. Roughly speaking, one can observe that all techniques have presented similar behavior concerning variations on the threshold value. In addition, the great majority of the datasets have been better described with a threshold greater or equal than $50 \%$, which means there might be an inferior bound for the feature selection problem. However, as the threshold increases, it does not imply the accuracy will also increase. Additionally, its is important to shed light over that BMOA 

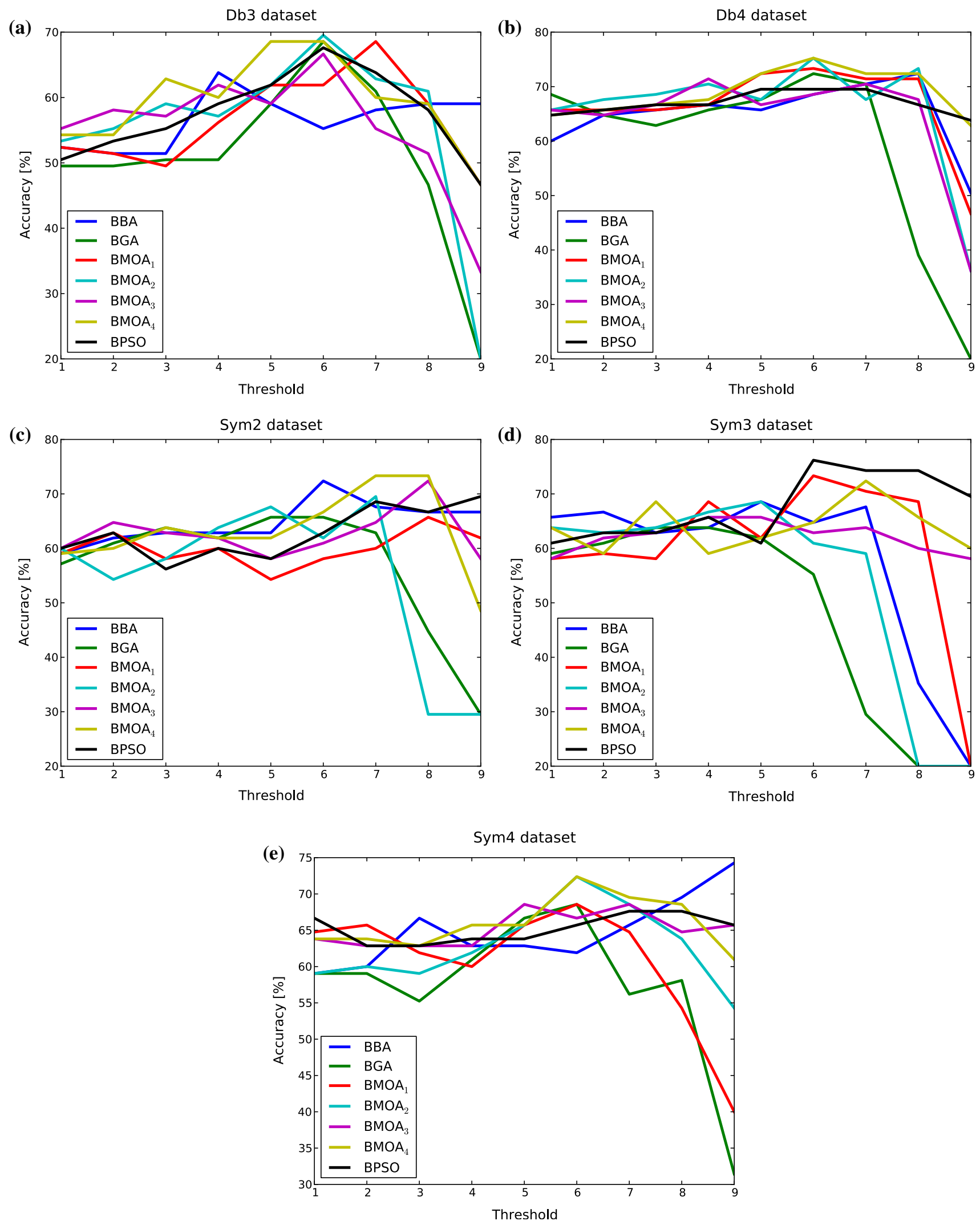

Fig. 7 Accuracy rates over the validating set considering Db3, Db4, Sym2, Sym3, and Sym4 datasets 
Table 5 F-measure, precision and recall rates over the testing set considering BMOA variants

\begin{tabular}{|c|c|c|c|c|c|c|c|c|c|c|c|c|c|c|c|c|c|c|c|c|}
\hline & \multicolumn{5}{|c|}{$\mathrm{BMOA}_{1}$} & \multicolumn{5}{|c|}{$\mathrm{BMOA}_{2}$} & \multicolumn{5}{|c|}{$\mathrm{BMOA}_{3}$} & \multicolumn{5}{|c|}{$\mathrm{BMOA}_{4}$} \\
\hline & A & B & C & D & E & A & B & $\mathrm{C}$ & D & E & A & B & $\mathrm{C}$ & D & E & A & B & $\mathrm{C}$ & D & E \\
\hline \multicolumn{21}{|c|}{ F-measure } \\
\hline Coif2 & 64 & 80 & 68 & 52 & 92 & 67 & 70 & 70 & 54 & 90 & 63 & 71 & 70 & 46 & 92 & 63 & 64 & 72 & 55 & 89 \\
\hline Coif3 & 79 & 77 & 58 & 50 & 88 & 68 & 69 & 70 & 63 & 90 & 71 & 75 & 65 & 67 & 95 & 83 & 84 & 75 & 67 & 95 \\
\hline Coif4 & 84 & 90 & 59 & 51 & 95 & 69 & 72 & 62 & 64 & 91 & 77 & 88 & 62 & 58 & 95 & 83 & 84 & 61 & 50 & 97 \\
\hline Db2 & 73 & 67 & 56 & 50 & 85 & 68 & 60 & 50 & 45 & 85 & 84 & 90 & 49 & 46 & 87 & 75 & 72 & 49 & 49 & 90 \\
\hline Db3 & 78 & 86 & 46 & 45 & 94 & 81 & 78 & 56 & 59 & 91 & 74 & 70 & 55 & 54 & 92 & 70 & 73 & 60 & 52 & 92 \\
\hline Db4 & 77 & 71 & 64 & 48 & 83 & 85 & 73 & 64 & 48 & 87 & 81 & 71 & 62 & 51 & 88 & 76 & 78 & 66 & 54 & 87 \\
\hline Sym2 & 73 & 66 & 51 & 48 & 90 & 73 & 59 & 49 & 40 & 87 & 81 & 77 & 46 & 49 & 86 & 77 & 80 & 54 & 56 & 85 \\
\hline Sym3 & 74 & 68 & 45 & 54 & 90 & 63 & 63 & 35 & 41 & 87 & 63 & 63 & 42 & 45 & 89 & 78 & 72 & 50 & 52 & 87 \\
\hline Sym4 & 67 & 71 & 59 & 50 & 84 & 68 & 68 & 65 & 53 & 84 & 67 & 72 & 57 & 46 & 93 & 65 & 66 & 65 & 57 & 85 \\
\hline \multicolumn{21}{|c|}{ Precision } \\
\hline Coif2 & 69 & 80 & 63 & 54 & 90 & 67 & 67 & 67 & 64 & 88 & 57 & 77 & 64 & 55 & 93 & 63 & 74 & 64 & 60 & 85 \\
\hline Coif3 & 76 & 81 & 59 & 47 & 93 & 66 & 76 & 70 & 61 & 90 & 69 & 76 & 72 & 64 & 94 & 77 & 89 & 76 & 67 & 97 \\
\hline Coif4 & 81 & 88 & 61 & 52 & 97 & 65 & 75 & 73 & 56 & 96 & 75 & 90 & 61 & 59 & 97 & 77 & 89 & 59 & 54 & 97 \\
\hline Db2 & 73 & 70 & 59 & 50 & 78 & 73 & 60 & 54 & 41 & 84 & 81 & 88 & 57 & $\mathbf{5 0}$ & 78 & 76 & 71 & 52 & 48 & 88 \\
\hline Db3 & 88 & 92 & 55 & 37 & 91 & 83 & 79 & 62 & 51 & 96 & 83 & 74 & 60 & 46 & 88 & 79 & 70 & 63 & 49 & 90 \\
\hline $\mathrm{Db} 4$ & 75 & 62 & 69 & 54 & 86 & 84 & 70 & 58 & 60 & 87 & 78 & 72 & 54 & 62 & 93 & 79 & 70 & 62 & 58 & 96 \\
\hline Sym2 & 70 & 62 & 56 & 50 & 90 & 70 & 61 & 52 & 40 & 84 & 83 & 75 & 50 & 46 & 89 & 71 & 74 & 64 & 59 & 84 \\
\hline Sym3 & 72 & 66 & 52 & 52 & 90 & 67 & 63 & 33 & 43 & 84 & 71 & 55 & 44 & 46 & 87 & 74 & 68 & 59 & 54 & 84 \\
\hline Sym4 & 67 & 69 & 58 & 50 & 89 & 69 & 66 & 60 & 56 & 89 & 70 & 71 & 51 & 48 & 96 & 62 & 68 & 58 & 65 & 86 \\
\hline \multicolumn{21}{|l|}{ Recall } \\
\hline Coif2 & 60 & 80 & 73 & 50 & 93 & 67 & 73 & 73 & 47 & 93 & 70 & 67 & 77 & 40 & 90 & 63 & 57 & 83 & 50 & 93 \\
\hline Coif3 & 83 & 73 & 57 & 53 & 83 & 70 & 63 & 70 & 67 & 90 & 73 & 73 & 60 & 70 & 97 & 90 & 80 & 73 & 67 & 93 \\
\hline Coif4 & 87 & 93 & 57 & 50 & 93 & 73 & 70 & 53 & 73 & 87 & 80 & 87 & 63 & 57 & 93 & 90 & 80 & 63 & 47 & 97 \\
\hline Db2 & 73 & 63 & 53 & 50 & 93 & 63 & 60 & 47 & 50 & 87 & 87 & 93 & 43 & 43 & 97 & 73 & 73 & 47 & $\mathbf{5 0}$ & 93 \\
\hline Db3 & 70 & 80 & 40 & 57 & 97 & 80 & 77 & 50 & 70 & 87 & 67 & 67 & 50 & 63 & 97 & 63 & 77 & 57 & 57 & 93 \\
\hline Db4 & 80 & 83 & 60 & 43 & 80 & 87 & 77 & 70 & 40 & 87 & 83 & 70 & 73 & 43 & 83 & 73 & 87 & 70 & 50 & 80 \\
\hline Sym2 & 77 & 70 & 47 & 47 & 90 & 77 & 57 & 47 & 40 & 90 & 80 & 80 & 43 & 53 & 83 & 83 & 87 & 47 & 53 & 87 \\
\hline Sym3 & 77 & 70 & 40 & 57 & 90 & 60 & 63 & 37 & 40 & 90 & 57 & 73 & 40 & 43 & 90 & 83 & 77 & 43 & 50 & 90 \\
\hline Sym4 & 67 & 73 & 60 & $\mathbf{5 0}$ & 80 & 67 & 70 & 70 & $\mathbf{5 0}$ & 80 & 63 & 73 & 63 & 43 & 90 & 67 & 63 & 73 & $\mathbf{5 0}$ & 83 \\
\hline
\end{tabular}

Bold values indicate the most accurate techniques variants obtained the best results in four out nine datasets, and also they achieved the same recognition rate as BPSO and BBA for Sym3 and Sym4 datasets, respectively.

If we consider Coif2 dataset, for instance, $\mathrm{BMOA}_{1}$ was the best technique with $80.95 \%$ of accuracy (considering a threshold of 50\%), as displayed in Fig. 6a. In addition, $\mathrm{BMOA}_{3}$ selected the $60 \%$ of the features that maximized the classification rate for Coif3 dataset (Fig. 6b). Finally, for Coif4 dataset, $\mathrm{BMOA}_{2}$ and BBA were the best performers with $77.14 \%$ of accuracy and a threshold equal to $70 \%$. In case of $\mathrm{Db}$ datasets, for $\mathrm{Db} 2, \mathrm{BMOA}_{3}$ and $\mathrm{BMOA}_{4}$ achieved the same accuracy rates, but with a different threshold: 60 and $70 \%$, respectively. For Db3 dataset, $\mathrm{BMOA}_{2}$ achieved $69.52 \%$ of recognition rate considering a threshold of $60 \%$. For Db4 dataset, $\mathrm{BMOA}_{4}$ maximized the accuracy measure with a threshold of
$60 \%$, reaching $75.23 \%$. Considering Sym2 dataset, $\mathrm{BMOA}_{4}$ was the best performer achieving $73.33 \%$ of accuracy (threshold of 70\%), and for Sym3 and Sym4, BPSO and BBA achieved the best accuracy rates of 76.19 and $74.28 \%$, respectively.

From Tables 3 and 4, it is possible to observe the proposed $\mathrm{BMOA}_{4}$ has been the most accurate technique in five out nine datasets, being them: Coif3, Db4, Sym2, Sym3 and Sym4. These results show us that the $\mathrm{BMOA}_{4}$ interaction mechanism provides better convergence rates than the other BMOA variants. Among the other algorithms, BPSO was the best performer in four out of nine datasets. It also achieved a great result over the Coif3 dataset with accuracy equal to $80 \%$, being slightly less accurate than $\mathrm{BMOA}_{4}$. Nevertheless, $\mathrm{BMOA}_{4}$ has selected less features than BPSO. 
Table 6 F-measure, precision and recall rates over the testing set considering BBA, BGA, BPSO and MI techniques

\begin{tabular}{|c|c|c|c|c|c|c|c|c|c|c|c|c|c|c|c|c|c|c|c|c|}
\hline & \multicolumn{5}{|c|}{ BBA } & \multicolumn{5}{|c|}{ BGA } & \multicolumn{5}{|c|}{ BPSO } & \multicolumn{5}{|l|}{ MI } \\
\hline & A & B & $\mathrm{C}$ & D & E & A & B & $\mathrm{C}$ & D & $\mathrm{E}$ & A & B & $\mathrm{C}$ & $\mathrm{D}$ & E & A & B & $\mathrm{C}$ & D & E \\
\hline \multicolumn{21}{|c|}{$F$-measure } \\
\hline Coif2 & 66 & 73 & 63 & 50 & 90 & 64 & 76 & 72 & 56 & 90 & 64 & 67 & 64 & 45 & 88 & 62 & 88 & 62 & 67 & 93 \\
\hline Coif3 & 74 & 72 & 63 & 58 & 93 & 80 & 84 & 66 & 67 & 91 & 82 & 85 & 72 & 67 & 91 & 78 & 85 & 80 & 67 & 93 \\
\hline Coif4 & 73 & 82 & 68 & 68 & 98 & 73 & 84 & 73 & 57 & 94 & 80 & 90 & 60 & 50 & 94 & 74 & 86 & 65 & 49 & 97 \\
\hline $\mathrm{Db} 2$ & 70 & 68 & 58 & 51 & 95 & 72 & 77 & 58 & 40 & 86 & 65 & 74 & 54 & 47 & 90 & 60 & 70 & 60 & 50 & 78 \\
\hline Db4 & 68 & 60 & 55 & 50 & 90 & 85 & 88 & 48 & 57 & 95 & 70 & 71 & 52 & 41 & 94 & 68 & 85 & 59 & 38 & 91 \\
\hline Db3 & 75 & 79 & 51 & 27 & 81 & 88 & 73 & 57 & 38 & 79 & 80 & 77 & 57 & 39 & 83 & 65 & 56 & 45 & 50 & 91 \\
\hline Sym2 & 75 & 64 & 39 & 48 & 92 & 70 & 55 & 54 & 56 & 86 & 76 & 79 & 52 & 39 & 87 & 71 & 77 & 50 & 50 & 79 \\
\hline Sym3 & 75 & 74 & 47 & 38 & 85 & 62 & 50 & 36 & 46 & 78 & 78 & 70 & 55 & 55 & 83 & 58 & 62 & 50 & 50 & 88 \\
\hline Sym4 & 76 & 80 & 59 & 24 & 91 & 63 & 68 & 60 & 55 & 85 & 61 & 68 & 58 & 51 & 84 & 64 & 64 & 54 & 60 & 89 \\
\hline \multicolumn{21}{|c|}{ Precision } \\
\hline Coif2 & 70 & 73 & 58 & 56 & 87 & 73 & 76 & 61 & 65 & 87 & 62 & 73 & 62 & 48 & 84 & 87 & 70 & 70 & 47 & 90 \\
\hline Coif3 & 71 & 75 & 63 & 56 & 96 & 74 & 86 & 75 & 62 & 96 & 85 & 81 & 75 & 65 & 93 & 83 & 77 & 67 & 80 & 93 \\
\hline Coif4 & 73 & 78 & 71 & 71 & 96 & 69 & 86 & 69 & 61 & 96 & 78 & 90 & 60 & 51 & 96 & 87 & 80 & 37 & 67 & 93 \\
\hline $\mathrm{Db} 2$ & 74 & 69 & 53 & 56 & 91 & 75 & 71 & 59 & 40 & 89 & 72 & 69 & 52 & 52 & 88 & 70 & 70 & 50 & 40 & 93 \\
\hline Db3 & 73 & 60 & 53 & 47 & 93 & 84 & 93 & 54 & 51 & 94 & 74 & 66 & 54 & 43 & 91 & 77 & 73 & 57 & 37 & 97 \\
\hline $\mathrm{Db} 4$ & 74 & 77 & 48 & 33 & 76 & 82 & 80 & 50 & 50 & 76 & 80 & 75 & 51 & 48 & 83 & 87 & 47 & 67 & 30 & 70 \\
\hline Sym2 & 81 & 66 & 41 & 43 & 90 & 67 & 60 & 58 & 50 & 89 & 73 & 76 & 52 & 48 & 84 & 73 & 77 & 40 & 53 & 87 \\
\hline Sym3 & 71 & 69 & 47 & 45 & 86 & 64 & 45 & 40 & 43 & 88 & 74 & 67 & 60 & 57 & 83 & 60 & 77 & 50 & 33 & 93 \\
\hline Sym4 & 73 & 80 & 51 & 32 & 96 & 63 & 69 & 54 & 60 & 86 & 62 & 66 & 54 & 52 & 92 & 70 & 60 & 67 & 50 & 80 \\
\hline \multicolumn{21}{|l|}{ Recall } \\
\hline Coif2 & 63 & 73 & 70 & 46 & 93 & 56 & 76 & 86 & 50 & 93 & 66 & 63 & 66 & 43 & 93 & 72 & 78 & 66 & 55 & 92 \\
\hline Coif3 & 76 & 70 & 63 & 60 & 90 & 86 & 83 & 60 & 73 & 86 & 80 & 90 & 70 & 70 & 90 & 81 & 81 & 73 & 73 & 93 \\
\hline Coif4 & 73 & 86 & 66 & 66 & 100 & 76 & 83 & 76 & 53 & 93 & 83 & 90 & 60 & 50 & 93 & 80 & 83 & 47 & 56 & 95 \\
\hline Db2 & 67 & 67 & 63 & 47 & 100 & 70 & 83 & 57 & 40 & 83 & 60 & 80 & 57 & 43 & 93 & 65 & 70 & 55 & 44 & 85 \\
\hline Db3 & 63 & 60 & 57 & 53 & 87 & 87 & 83 & 43 & 63 & 97 & 67 & 77 & 50 & 40 & 97 & 72 & 79 & 58 & 37 & 94 \\
\hline Db4 & 77 & 80 & 53 & 23 & 87 & 93 & 67 & 67 & 30 & 83 & 80 & 80 & 63 & 33 & 83 & 74 & 51 & 54 & 38 & 79 \\
\hline Sym2 & 70 & 63 & 37 & 53 & 93 & 73 & 50 & 50 & 63 & 83 & 80 & 83 & 53 & 33 & 90 & 72 & 77 & 44 & 52 & 83 \\
\hline Sym3 & 80 & 80 & 47 & 33 & 83 & 60 & 57 & 33 & 50 & 70 & 83 & 73 & $\mathbf{5 0}$ & 53 & 83 & 59 & 69 & 50 & 40 & 90 \\
\hline Sym4 & 80 & 80 & 70 & 20 & 87 & 63 & 67 & 67 & 50 & 83 & 60 & 70 & 63 & 50 & 77 & 67 & 62 & 60 & 55 & 84 \\
\hline
\end{tabular}

Bold values indicate the most accurate techniques
Figure 2 displays the statistical test concerning the accuracy results. Clearly, one can observe the proposed BMOA approaches (i.e., $\mathrm{BMOA}_{4}$ and $\mathrm{BMOA}_{3}$ ) have been placed as the two top best techniques (from right to left), though all techniques have been considered similar to each other, except the baseline provided by OPF (i.e., without feature selection).

Similarly, Figs. 3, 4, and 5 depict the statistical tests concerning the F-measure, precision and recall results. Notice all performance measures placed the proposed approaches as the best ones, tough all being similar to each other concerning the statistical test, excepting the baseline provided by OPF. Roughly speaking, we can argue the proposed approaches are suitable for feature selection, and the neighborhood information can really improve the results (Figs. 6, 7).

In regard to the $F$ - measure results (Table 5), which is the harmonic average between precision and recall measures, BMOA variants have achieved the highest values for classes A, B, and C, since such classes are well separated by kernel machines in general (please, refer to [14]). The proposed $\mathrm{BMOA}_{4}$ has been the one with the highest accuracy over class $\mathrm{D}$, followed by $\mathrm{BMOA}_{1}$ and $\mathrm{BMOA}_{2}$ that achieved the best results over classes B (a tie with BPSO can be observed) and A, respectively. In addition, BBA has obtained the best accuracy considering class D. For the sake of comparison purposes, Table 6 displays the F-measure values concerning the techniques compared in this work. 


\section{Concluding remarks}

In this work, we carried the problem of EEG signal classification by means of four variants of the magnetic optimization algorithm, being two of them proposed in this work. In addition, three well-known metaheuristic algorithms were considered in this study, namely particle swarm optimization, binary bat algorithm, and genetic algorithm.

The proposed $\mathrm{BMOA}_{4}$ variant has prevailed in terms of effectiveness (accuracy, precision, recall, and F-measure) measures considering the great majority of datasets, as well as in terms of the number of selected features. In special, $\mathrm{BMOA}_{4}$ recognition rate over the features extracted via Coif-3 wavelets has shown very satisfactory levels of performance (with accuracy equal to $81 \%$ ). Besides, $\mathrm{BMOA}_{4}$ has always prevailed over the other BMOA-based methods in terms of the discrimination power between classes $\mathrm{C}, \mathrm{D}$, and $\mathrm{E}$.

It is also worth noting the main idea of this work is to show the importance in considering distinct neighborhood information when dealing with metaheuristic techniques. The proposed approaches were validated in the context of feature selection purposes concerning the task of epileptic identification by means of EEG signals. Although state-of-the-art results were not achieved, BMOA approaches seemed to be very much suitable to the problem, as well as they can also be applied to different other applications.

Acknowledgements LAMP and JPP are grateful to FAPESP Grants \#2011/14094-1, \#2009/16206-1, and \#2014/16250-9, respectively, and also CNPq Grants \#303182/2011-3, \#470571/2013-6, and $\# 306166 / 2014-3$. The ALVC and CAML also acknowledge the sponsorship from CNPq via Grants \#475406/2010-9, \#304603/20120, 308816/2012-9, and \#303182/2011-3. VHCA acknowledges CNPq for the Grants \#470501/2013-8 and \#301928/2014-2.

\section{Compliance with ethical standards}

Conflicts of interest The authors declare no conflict of interest.

\section{References}

1. Andrzejak RG, Lehnertz K, Mormann F, Rieke C, David P, Elger CE (2001) Indications of nonlinear deterministic and finite dimensional structures in time series of brain electrical activity: dependence on recording region and brain state. Phys Rev E Stat Nonlinear Soft Matter Phys 64:061907-1-061907-6

2. Andrzejak RG, Widman G, Lehnertz K, Rieke C, David P, Elger CE (2001) The epileptic process as nonlinear deterministic dynamics in a stochastic environment: an evaluation on mesial temporal lobe epilepsy. Epilepsy Res 44:129-140

3. Browne TR, Holmes GL (2003) Handbook of Epilepsy. Lippincott Williams \& Wilkins, Philadelphia
4. Chang BS, Lowenstein DH (2003) Epilepsy. N Engl J Med 349:1257-1266

5. Demšar J (2006) Statistical comparisons of classifiers over multiple data sets. J Mach Learn Res 7:1-30

6. Firpi HA, Goodman E (2004) Swarmed feature selection. In: Proceedings of the 33rd applied imagery pattern recognition workshop, IEEE Computer Society, Washington, DC, USA, pp $112-118$

7. Gandhi T, Panigrahi BK, Anan S (2011) A comparative study of wavelet families for EEG signal classification. Neurocomputing 74(17):3051-3057

8. Gotman J (1982) Automatic recognition of epileptic seizures in the EEG. Electroencephalogr Clin Neurophysiol 54:530-540

9. Guyon I, Elisseeff A (2003) An introduction to variable and feature selection. J Mach Learn Res 3:1157-1182

10. Guyon I, Gunn S, Nikravesh M, Zadeh LA (2006) Feature extraction: foundations and applications. Springer, Berlin

11. Hazarika N, Chen JZ, Tsoi AC, Sergejew A (1997) Classification of EEG signals using the wavelet transform. Signal Process 59:61-72

12. Kaveh A, Talatahari S (2010) A novel heuristic optimization method: charged system search. Acta Mech 213(3):267-289

13. Koza JR (1992) Genetic programming: on the programming of computers by means of natural selection. MIT Press, Cambridge

14. Lima CAM, Coelho ALV (2011) Kernel machines for epilepsy diagnosis via EEG signal classification: a comparative study. Artif Intell Med 53:83-95

15. Lima CAM, Coelho ALV, Chagas S (2009) Automatic EEG signal classification for epilepsy diagnosis with relevance vector machines. Expert Syst Appl 36:10054-10059

16. Lima CAM, Coelho ALV, Eisencraft M (2010) Tackling EEG signal classification with least squares support vector machines: a sensitivity analysis study. Comput Biol Med 40:705-714

17. Lin S-W, Ying K-C, Chen S-C, Lee Z-J (2008) Particle swarm optimization for parameter determination and feature selection of support vector machines. Expert Syst Appl 35(4):1817-1824

18. Mirjalili S, Mohd Hashim SZ (2011) BMOA: binary magnetic optimization algorithm. In: 3rd IEEE international conference on machine learning and computing, Singapore, vol 1, pp 201-206

19. Nakamura RYM, Pereira LAM, Costa KA, Rodrigues D, Papa JP, Yang X-S (2012) BBA: a binary bat algorithm for feature selection. In: Proceedings of the XXV SIBGRAPI-conference on graphics, patterns and images, pp 291-297

20. Nemenyi P (1963) Distribution-free multiple comparisons. Princeton University, Princeton

21. Nunes TM, Coelho ALV, Lima CAM, Papa JP, de Albuquerque VHC (2014) EEG signal classification for epilepsy diagnosis via optimum path forest-a systematic assessment. Neurocomputing 136:103-123

22. Ocak H (2008) Optimal classification of epileptic seizures in EEG using wavelet analysis and genetic algorithm. Signal Process 88:1858-1867

23. Papa JP, Falcão AX, Albuquerque VHC, Tavares JMRS (2012) Efficient supervised optimum-path forest classification for large datasets. Pattern Recognit 45(1):512-520

24. Papa JP, Falcão AX, Suzuki CTN (2009) Supervised pattern classification based on optimum-path forest. Int J Imaging Syst Technol 19(2):120-131

25. Rodrigues D, Pereira LAM, Nakamura RYM, C KAP, Yang X-S, Souza AN, Papa JP (2014) A wrapper approach for feature selection based on bat algorithm and optimum-path forest. Expert Syst Appl 41(5):2250-2258

26. Subasi A (2005) Epileptic seizure detection using dynamic wavelet network. Expert Syst Appl 28:701-711 
27. Subasi A (2007) EEG signal classification using wavelet feature extraction and a mixture of expert model. Expert Syst Appl 32:1084-1093

28. Subasi A, Ercelebi E (2005) Classification of EEG signals using neural network and logistic regression. Comput Methods Programs Biomed 78:87-99

29. Tang YY (2009) Wavelet Theory Approach to Pattern Recognition. World Scientific Publishing, Singapore

30. Tayarani MH, Akbarzadeh-Totonchi MR (2008) Magnetic optimization algorithms a new synthesis. In: IEEE congress on evolutionary computation, IEEE, pp 2659-2664

31. Tzallas AT, Tsipouras MG, Fotiadis DI (2007) Automatic seizure detection based on time-frequency analysis and artificial neural networks. Comput Intell Neurosci 2007:80510-1-80510-13

32. Tzallas AT, Tsipouras MG, Fotiadis DI (2009) Epileptic seizure detection in EEGs using time-frequency analysis. IEEE Trans Inf Technol Biomed 13:703-710
33. Übeyli ED (2008) Wavelet/mixture of experts network structure for EEG signals classification. Expert Syst Appl 34:1954-1962

34. Übeyli ED (2009) Combined neural network model employing wavelet coefficients for EEG signals classification. Digit Signal Process 19:297-308

35. Übeyli ED (2009) Statistics over features: EEG signals analysis. Comput Biol Med 39:733-741

36. Walnut DF (2004) An introduction to wavelet analysis. Birkhäuser, Basel

37. Willi-Hans S (2011) The nonlinear workbook, 5th edn. World Scientific, Singapore

38. Yang H, Du Q (2011) Particle swarm optimization-based dimensionality reduction for hyperspectral image classification. In: IEEE international geoscience and remote sensing symposium, pp 2357-2360

39. Yusta SC (2009) Different metaheuristic strategies to solve the feature selection problem. Pattern Recognit Lett 30:525-534 